\title{
Monitoring of a Wind Turbine Rotor using a Multi-blade Coordinate Framework
}

\author{
Henriksen, Lars Christian; Niemann, Hans Henrik; Poulsen, Niels Kjølstad
}

Published in:

System Identification

Link to article, DOI:

10.3182/20120711-3-BE-2027.00068

Publication date:

2012

Document Version

Publisher's PDF, also known as Version of record

Link back to DTU Orbit

Citation $(A P A)$ :

Henriksen, L. C., Niemann, H. H., \& Poulsen, N. K. (2012). Monitoring of a Wind Turbine Rotor using a Multiblade Coordinate Framework. In System Identification (Vol. 16, pp. 374-379). International Federation of Automatic Control. IFAC Proceedings Volumes (IFAC-PapersÓnline) https://doi.org/10.3182/20120711-3-BE2027.00068

\section{General rights}

Copyright and moral rights for the publications made accessible in the public portal are retained by the authors and/or other copyright owners and it is a condition of accessing publications that users recognise and abide by the legal requirements associated with these rights.

- Users may download and print one copy of any publication from the public portal for the purpose of private study or research.

- You may not further distribute the material or use it for any profit-making activity or commercial gain

- You may freely distribute the URL identifying the publication in the public portal 


\title{
Monitoring of a Wind Turbine Rotor using a Multi-blade Coordinate Framework ${ }^{\star}$
}

\author{
Lars Christian Henriksen* Hans Henrik Niemann ** \\ Niels Kjølstad Poulsen ${ }^{* *}$ \\ * Wind Energy Division, Risø National Laboratory for Sustainable \\ Energy, Technical University of Denmark, (e-mail: larh@risoe.dtu.dk) \\ ** Dept. of Electrical Engineering, Technical University of Denmark, \\ (e-mail:hhn@elektro.dtu.dk) \\ *** Dept. of Informatics and Mathematical Modelling, Technical \\ University of Denmark, (e-mail: nkp@imm.dtu.dk)
}

\begin{abstract}
In this paper a method to detect asymmetric faults in a wind turbine rotor is presented. The paper describes how fault diagnosis using an observer-based residual generator approach is able to distinguish between the nominal and faulty case by the injection of e.g. a sinusoidal excitation signal into the system. In the case of a wind turbine, an excitation signal is automatically generated by the rotation of the rotor in a turbulent wind field. Using the multi-blade coordinate transformation, the detection of asymmetries in the rotor of the wind turbine is greatly improved.
\end{abstract}

Keywords: Fault Detection and Diagnosis; Mechanical and Aerospace

\section{INTRODUCTION}

In the pursuit of an improved wind turbine operation economy, condition monitoring, fault diagnosis and fault tolerant control are important tools that can be used to detect faults and determine the prober action. E.g. stop the wind turbine immediately and wait for maintenance or continue operation with a reconfigured controller, which accommodate the changes detected on the wind turbine.

The wind turbine can be subjected to various faults on various components: Using continuous wavelet transformation (CWT) Tsai et al. [2006] looked at blade damage detection and Watson et al. [2010] have investigated generator and drive train faults with CWT analysis of the generator power output sensor. Rotor condition monitoring for a number of different blade-specific faults have been examined by Caselitz and Giebhardt [2005]. The works cited above all share the direct use of sensor analysis to detect faults. However, if the controller is able to suppress the effect of the fault, sensor-based detection techniques fall short and are not able to detect the fault.

Observer-based techniques for residual generation fault detection Frank and Ding [1994], Frank [1996] on the other hand, take both the sensors and control actions into account and are thus able to detect faults even in closed-loop operation. To further enhance the fault diagnosis, active measures can be taken. In e.g. Niemann [2006], Poulsen and Niemann [2009a,b] an auxiliary signal is injected into the system to aid the fault detection. In Niemann and Poulsen [2008] both active and passive fault diagnosis is discussed. In the passive fault diagnosis, partial or full

\footnotetext{
* This work was supported in part by the CASED Project funded by grant DSF-09-063197 of the Danish Council for Strategic Research.
}

knowledge about an uncontrolled disturbance is used for fault diagnosis.

Observer-based fault detection techniques have previously been applied on wind turbines e.g. Odgaard et al. [2009], Odgaard and Stoustrup [2009, 2010] and Wei et al. [2008], Wei and Verhaegen [2011]. However, the mentioned references use a different evaluation method of the residuals to determine if a fault is present and do not explicitly exploit the auxiliary signal automatically generated by the rotation of the rotor. For an overview of various fault detection and condition monitoring algorithms applied to wind turbines Hameed et al. [2009] can be consulted.

In this paper a wind turbine is subjected to fault diagnosis and two cases are presented: A nominal case and fault case where faults on the turbine rotor are included. These faults are offset in pitch actuator, offset on edgewise and flapwise strang gauge sensors for each blade. An extended Kalman filter will be used as the observer-based residual generator and the residuals will be analyzed by a CUSUM test to detect faults. The rotation of the wind turbine acts as a natural injection of a periodic signal, which can be detected in the residuals in the faulty case. In the presented example, the wind turbine operates in partial load conditions and the rotor speed thus varies as a function of wind speed to maximize power capture. The varying rotor speed result in an injected signal with varying frequency. The presented detection method is able to handle the varying rotor speed and results show good detection performance even in the presence of varying rotor speed.

Simulations are performed in the multi-body aero-servoelastic software HAWC2 Larsen and Hansen [2007] developed by Ris $\varnothing$ DTU. The wind turbine used in the 
simulations is the $5 \mathrm{MW}$ reference wind turbine defined in Jonkman et al. [2009]. The extended Kalman filter bases its estimations on a control design model, which does not include all the degrees of freedom included in HAWC2. A significant difference is blade flexibility resulting in blade deformation giving steady state properties different from those predicted by the control design model. This difference leads to an offset in the estimated wind speed compared to the wind speed in HAWC2. Accordingly, the nominal case is not entirely nominal and residuals are expected to have mean values different from zero even in the nominal case. The task is then to determine the nominal conditions and detect if the residuals differ from the nominal values.

The multi-blade coordinate (MBC) transformation also denoted the Coleman transformation Coleman and Feingold [1958] enables a time-varying system to be transformed to a time-invariant system, when the rotor of the wind turbine, helicopter etc. is assumed isotropic, i.e. symmetric. If asymmetries do occur, the MBC transformed system will not be time-invariant. This property can be exploited to ease the fault detection. By transforming the wind turbine model coordinates to the multi-blade coordinates, the nominal case will have zero-mean residuals and the faulty case will have non-zero-mean residuals. Furthermore, the strength of $\mathrm{MBC}$ is that time-varying system becomes time-invariant and that a individual pitching controller is easily described. MBC for dynamical analysis of the wind turbine is a strong tool and e.g. Hansen [2003] and Bir [2008] discusses the subject in much greater depth.

The outline of this paper is as follows: The multi-blade coordinate transformation and its application to state space models is explained in Section 2. The wind turbine model presented in Section 3 is used by the extended Kalman filter in Section 4. In Section 5 implemented fault diagnosis method is discussed and simulations demonstrating the potential of the proposed method are shown in Section 6 . Finally conclusions are drawn in Section 7.

\section{MULTI-BLADE COORDINATE TRANSFORMATION}

In this section the fundamentals of the multi-blade coordinate (MBC) transformation are introduced in the first subsection. Following is a subsection describing how the MBC transformation is applied on the state space model. Unfortunately, space constraints prevent more details to be presented in this work and Henriksen et al. [2011a,b] can be investigated for further details regarding the transformation from rotating frame to fixed frame. In Henriksen et al. [2011a] a discussion, on how asymmetric rotating frame signals result in time-varying fixed frame transformed coordinates, is also presented.

\section{1 $M B C$ fundamentals}

The MBC transformation enables the transformation from a rotating frame of reference to a fixed frame of reference. The azimuth angle $\phi_{i}$ of blades $i=1, \ldots, n_{\text {blades }}$, assuming constant rotor speed $\Omega$ and equal angular spacing between the blades, is given by

$$
\phi_{i}=\phi_{0}+\Omega t-(i-1) \pi / n_{\text {blades }}
$$

and renders the MBC transformation a function of time $t$ rather than the azimuth angle $\phi$. The azimuth angles can be combined in a vector, which for a 3-bladed rotor is $\phi=\left[\begin{array}{lll}\phi_{1} & \phi_{2} & \phi_{3}\end{array}\right]^{T}$. The temporal argument of states and transformation matrices in the following has been omitted to simplify notation. The rotating frame coordinates $\boldsymbol{q}$ and the fixed frame coordinates $\boldsymbol{q}^{\boldsymbol{f}}$ have the following relationship

$$
\boldsymbol{q}=\mathbf{M}^{-1} \boldsymbol{q}^{f}
$$

where the element of $\boldsymbol{q}$ are denoted $q_{i}$ for blades $i=1,2$ and 3. The elements of $\boldsymbol{q}^{f}$ are denoted $q_{i}^{f}$ for the fixed frame coordinates $i=0, c$ and $s$, corresponding to the mean, cosine and sine part of the fixed frame coordinates, respectively. The $\mathrm{MBC}$ transformation matrices are

$$
\mathbf{M}=\left[\begin{array}{c}
\frac{1}{3} \mathbf{1}^{T} \\
\frac{2}{3} \cos \phi^{T} \\
\frac{2}{3} \sin \phi^{T}
\end{array}\right], \quad \mathbf{M}^{-1}=\left[\begin{array}{c}
\mathbf{1}^{T} \\
\cos \phi^{T} \\
\sin \phi^{T}
\end{array}\right]^{T},
$$

where $\mathbf{1}=\left[\begin{array}{lll}1 & 1 & 1\end{array}\right]^{T}$ and $\cos \phi=\left[\begin{array}{lll}\cos \phi_{1} & \cos \phi_{2} & \cos \phi_{3}\end{array}\right]^{T}$ etc.

\subsection{The $M B C$ transformation applied on a state space model}

A dynamic system in state space form can be expressed by an nonlinear ordinary differential equation vector function and a vector output function as

$$
\begin{aligned}
& \dot{\boldsymbol{x}}(t)=\boldsymbol{f}(\boldsymbol{x}(t), \boldsymbol{u}(t), t) \\
& \boldsymbol{y}(t)=\boldsymbol{g}(\boldsymbol{x}(t), \boldsymbol{u}(t), t)
\end{aligned}
$$

where states $\boldsymbol{x}$, inputs $\boldsymbol{u}$, outputs $\boldsymbol{y}$ and the vector functions $\boldsymbol{f}$ and $\boldsymbol{g}$ are all functions of time. In the following, the temporal arguments of states, inputs and outputs and vector functions have been omitted to simplify notation.

First order Taylor expansion around the linearization $(\overline{\boldsymbol{x}}, \overline{\boldsymbol{u}})$ yields

$$
\begin{aligned}
& \dot{\boldsymbol{x}}=\boldsymbol{f}(\overline{\boldsymbol{x}}, \overline{\boldsymbol{u}})+\mathbf{A}(\boldsymbol{x}-\overline{\boldsymbol{x}})+\mathbf{B}(\boldsymbol{u}-\overline{\boldsymbol{u}}) \\
& \boldsymbol{y}=\boldsymbol{g}(\overline{\boldsymbol{x}}, \overline{\boldsymbol{u}})+\mathbf{C}(\boldsymbol{x}-\overline{\boldsymbol{x}})+\mathbf{D}(\boldsymbol{u}-\overline{\boldsymbol{u}})
\end{aligned}
$$

where the system matrices $(\mathbf{A}, \mathbf{B}, \mathbf{C}, \mathbf{D})$ are functions of time. The linearization can be rewritten to

$$
\begin{array}{ll}
\dot{\boldsymbol{x}}=\mathrm{A} \boldsymbol{x}+\mathrm{B} \boldsymbol{u}+\boldsymbol{\delta}, & \boldsymbol{\delta}=\boldsymbol{f}(\overline{\boldsymbol{x}}, \overline{\boldsymbol{u}})-\mathrm{A} \overline{\boldsymbol{x}}-\mathrm{B} \overline{\boldsymbol{u}} \\
\boldsymbol{y}=\mathrm{C} \boldsymbol{x}+\mathrm{D} \boldsymbol{u}+\gamma, & \gamma=\boldsymbol{g}(\overline{\boldsymbol{x}}, \overline{\boldsymbol{u}})-\mathbf{C} \overline{\boldsymbol{x}}-\mathrm{D} \overline{\boldsymbol{u}}
\end{array}
$$

for typical linear control theory the pair $(\overline{\boldsymbol{x}}, \overline{\boldsymbol{u}})$ is chosen to be an equilibrium point (such that $\mathbf{0}=\boldsymbol{f}(\overline{\boldsymbol{x}}, \overline{\boldsymbol{u}})$ ), but the theory is also valid for other choices of $(\overline{\boldsymbol{x}}, \overline{\boldsymbol{u}})$.

The time-varying combined fixed and rotating frame system (3) can be transformed to a fixed frame time-invariant system where the states, inputs and outputs are transformed to the fixed frame of reference

$$
\boldsymbol{x}^{f}=\mathbf{M}_{\mathbf{x}} \boldsymbol{x} \text { and } \boldsymbol{u}^{\boldsymbol{f}}=\mathrm{M}_{\mathrm{u}} \boldsymbol{u} \text { and } \boldsymbol{y}^{f}=\mathrm{M}_{\mathrm{y}} \boldsymbol{y} .
$$

The MBC transformations gives the fixed frame system equations

$$
\begin{aligned}
& \dot{x}^{f}=\mathbf{A}^{\mathbf{f}} x^{f}+\mathbf{B}^{\mathbf{f}} u^{f}+\delta^{\mathbf{f}} \\
& y^{f}=\mathbf{C}^{\mathbf{f}} x^{f}+\mathbf{D}^{\mathbf{f}} u^{f}+\gamma^{f}
\end{aligned}
$$

The system matrices $\left(\mathbf{A}^{\mathbf{f}}, \mathbf{B}^{\mathbf{f}}, \mathbf{C}^{\mathbf{f}}, \mathbf{D}^{\mathbf{f}}\right)$ are time-invariant, as are the residual vectors $\left(\boldsymbol{\delta}^{\boldsymbol{f}}, \boldsymbol{\gamma}^{\boldsymbol{f}}\right)$ when rotating frame 
variables $\boldsymbol{q}$ within the state vector $\boldsymbol{x}$ have been averaged in the linearization point

$$
\overline{\boldsymbol{q}}=q_{0}^{f} \mathbf{1}
$$

The nonlinear time discrete state progress equation

$$
\boldsymbol{x}_{k+1}^{\boldsymbol{f}}=\underline{\boldsymbol{f}}^{\boldsymbol{f}}\left(\boldsymbol{x}_{k}^{\boldsymbol{f}}, \boldsymbol{u}_{k}^{\boldsymbol{f}}\right)=\boldsymbol{x}_{k}^{\boldsymbol{f}}+\int_{t_{k}}^{t_{k+1}} \dot{\boldsymbol{x}}^{\boldsymbol{f}}(\tau) d \tau
$$

used in Section 4 can be approximated by a linear description

$$
\begin{aligned}
\boldsymbol{x}_{k+1}^{f}=\underline{\mathrm{A}}^{\mathbf{f}} \boldsymbol{x}_{k}^{f}+\underline{\mathrm{B}}^{\mathbf{f}} \boldsymbol{u}_{k}^{f}+\underline{\delta}^{f}, \\
\underline{\delta}^{f}=\underline{\boldsymbol{f}}^{\boldsymbol{f}}\left(\overline{\boldsymbol{x}}^{f}, \overline{\boldsymbol{u}}^{\boldsymbol{f}}\right)-\underline{\mathrm{A}}^{\mathbf{f}} \overline{\boldsymbol{x}}^{f}-\underline{\mathbf{B}}^{\mathbf{f}} \overline{\boldsymbol{u}}^{f}
\end{aligned}
$$

and Henriksen and Poulsen [2010] gives further details regarding implementation.

\section{WIND TURBINE MODEL}

In this section the governing equations constituting the wind turbine model used by the fault detection algorithm is presented. Due to space constraint, the detailed description of the wind turbine model have been omitted and Henriksen et al. [2011a,b] can be consulted for more details regarding the wind turbine model. Instead, a small introduction to the variables included in the model is given in the following paragraphs.

The model for the aerodynamic forces affecting the blades of the rotor are calculated using blade element momentum (BEM) theory, where the free wind speed, the pitch angle of the blades and the relative motion of the individual blade elements are used to calculate the aerodynamic forces based based on the aerodynamic profile data for that particular element along the blade. Strain gauges on blades $i=1,2$ and 3 , denoted $Q_{n, i}^{S G}$ and $Q_{t, i}^{S G}$, are used to measure the blade root bending moments normal to and tangential to the rotor plane, respectively. The blades block the free wind speed and induced wind speeds normal to and tangential to the rotor plane are used in the BEM calculations. The dynamics of the induced wind speed normal to the rotor plane is modelled as a first order dynamical system with an equivalent induced wind speed $\bar{v}_{n, i}$ for blades $i=1,2$ and 3 .

The drive-train is modelled as two inertial rotating masses connected by a mass and spring. The blades of the rotor are in one end of the drive-train and the electrical generator is in the other end. The rotational speed of the rotor and generator is denoted $\Omega$ and $\Omega_{g}$, respectively. The torsional deflection in the drive-train is denoted $\phi_{\Delta}$. Inputs to the drive-train is the aerodynamic torque in the rotor end and the electro-mechanical torque of the generator in the other end. The fore-aft displacement of the tower, denoted $\psi_{t}$, is modelled as a mass, spring and damper system. The fore-aft velocity of the tower results in a relative wind speed, different from the free wind speed and through the aerodynamic equations the drive-train and tower fore-aft are coupled. The aerodynamic thrust is input to the foreaft tower displacement.

The generator torque actuator is modelled as a first order dynamical system with generator torque reference $Q_{g, r e f}$ as input and generator torque $Q_{g}$ as output. The electrical power output of the generator is denoted $P_{e}$. The pitch angle actuators for blades $i=1,2$ and 3 are modelled as second order dynamical systems with pitch angle reference $\theta_{\text {ref }, i}$ as input and pitch angle $\theta_{i}$ as output.

The wind turbulence model is modelled in the fixed frame and coupled to rest of the wind turbine model after it too has been transformed to the fixed frame via the multi-blade coordinate transformation. The wind speed in rotating frame is denoted $V_{i}$ for blades $i=1,2$ and 3 , which is the sum of a rotor wide mean wind speed $V_{m}$ and the individual turbulent wind speeds $V_{t, i}$ modelled as a second order dynamic systems driven by Gaussian distributed zero-mean noise. In the fixed frame the wind speed is denoted $V_{i}^{f}$ for the $i=0, c$, and $s$ components of the fixed frame coordinates.

The ordinary differential equations of the submodels in the rotating frame, are gathered in a state space ordinary differential equation and an output function in the form of (3) where $\boldsymbol{x}$ is the state vector, $\boldsymbol{u}$ is the input vector and $\boldsymbol{y}$ is the measurement vector. The vectors are comprised by the following variables

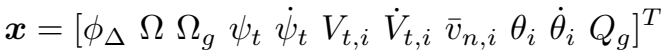

$$
\begin{aligned}
& \boldsymbol{u}=\left[\begin{array}{lll}
\theta_{\text {ref }, i} & Q_{g, r e f}
\end{array}\right]^{T}
\end{aligned}
$$

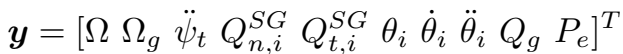

\section{EXTENDED KALMAN FILTER}

The extended Kalman filter (EKF) is used to estimate the states and generate the residuals used by the fault diagnosis algorithm.

The a posteriori estimate of the states with the time index $k \mid k$, meaning estimate at time $k$ given by the knowledge available at time $k$, is given by

$$
\begin{aligned}
\hat{\boldsymbol{x}}_{k \mid k}^{\boldsymbol{f}}=\hat{\boldsymbol{x}}_{k \mid k-1}^{\boldsymbol{f}}+ & \\
& \mathbf{L}_{k} \mathbf{M}_{\mathbf{y}, k}\left(\boldsymbol{y}_{i}-\boldsymbol{g}\left(\mathbf{M}_{\mathbf{x}, k}^{-1} \hat{\boldsymbol{x}}_{k \mid k-1}^{\boldsymbol{f}}, \mathbf{M}_{\mathbf{u}, k}^{-1} \boldsymbol{u}_{k}^{\boldsymbol{f}}\right)\right)
\end{aligned}
$$

Enabling an a priori estimate of the states with the time index $k+1 \mid k$, meaning estimate at time $k+1$ given knowledge available at time $k$, given by

$$
\hat{\boldsymbol{x}}_{k+1 \mid k}^{f}=\underline{\boldsymbol{f}}^{\boldsymbol{f}}\left(\hat{\boldsymbol{x}}_{k \mid k}^{f}, \boldsymbol{u}_{k}^{\boldsymbol{f}}\right)
$$

where the Kalman gain $\mathbf{L}_{k}$ and output error covariance $\boldsymbol{\Psi}_{k}$ are given by

$$
\begin{aligned}
\mathbf{L}_{k} & =\mathbf{P}_{k \mid k-1} \mathbf{C}_{k \mid k-1}^{\mathbf{f} T} \boldsymbol{\Psi}_{k}^{-1} \text { and } \\
\boldsymbol{\Psi}_{k} & =\mathbf{C}_{k \mid k-1}^{\mathbf{f}} \mathbf{P}_{k \mid k-1} \mathbf{C}_{k \mid k-1}^{\mathbf{f} T}+\mathbf{R}_{\mathbf{y}}
\end{aligned}
$$

respectively. The Kalman gain and output error covariance matrices are updated by the discrete time recursive Riccati equation

$$
\begin{aligned}
\mathbf{P}_{k \mid k} & =\mathbf{P}_{k \mid k-1}-\mathbf{L}_{k} \mathbf{C}_{k \mid k-1}^{\mathbf{f}} \mathbf{P}_{k \mid k-1} \\
\mathbf{P}_{k+1 \mid k} & =\mathbf{A}_{k \mid k}^{\mathbf{f}} \mathbf{P}_{k \mid k} \mathbf{A}_{k \mid k}^{\mathbf{f} T}+\mathbf{R}_{\mathbf{x}}
\end{aligned}
$$

The state estimate used by the full-state-feedback control algorithm can either be the a posteriori $\hat{\boldsymbol{x}}_{k \mid k}$ or the a priori $\hat{\boldsymbol{x}}_{k \mid k-1}$.

\section{FAULT DETECTION}

In the following section, the theory for an active/passive fault diagnosis (FD) method is presented. The method is based on the passive fault diagnosis found in e.g. Frank 
and Ding [1994], Niemann and Poulsen [2008] and on the active fault diagnosis found in e.g. Niemann [2006].

A system consisting of the transfer functions $\mathbf{G}_{\mathbf{e d}}, \mathbf{G}_{\mathbf{e d}}$, $\mathbf{G}_{\mathbf{e d}}$ and $\mathbf{G}_{\mathbf{e d}}$ is given by

$$
\boldsymbol{\Sigma}_{\mathbf{P}}:\left\{\begin{array}{l}
e=\mathbf{G}_{\mathrm{ed}}(p) d+\mathbf{G}_{\mathrm{eu}}(p) u \\
y=\mathbf{G}_{\mathrm{yd}}(p) d+\mathbf{G}_{\mathrm{yu}}(p) u
\end{array}\right.
$$

where the output vectors $\boldsymbol{e}$ and $\boldsymbol{y}$ are control objectives and measurements, respectively. The input signals $\boldsymbol{d}$ and $\boldsymbol{u}$ are input disturbance and control signal, respectively. The transfer functions are functions of the parameter vector $\boldsymbol{p}=\left[p_{1}, \ldots\right]^{T}$, where $\boldsymbol{p}=\mathbf{0}$ is the nominal case. Further, let the system be controlled by a stabilizing controller

$$
\boldsymbol{\Sigma}_{\mathbf{C}}:\{\boldsymbol{u}=\mathbf{K} \boldsymbol{y}
$$

The nominal system given by $\mathbf{G}_{\mathbf{y u}}(\mathbf{0})$ and the stabilizing controller $\mathbf{K}$ can be subjected to a co-prime factorization

$$
\begin{aligned}
\mathbf{G}_{\mathbf{y u}}(\mathbf{0}) & =\mathbf{N M}^{-1}=\tilde{\mathbf{M}}^{-1} \tilde{\mathbf{N}} \\
\mathbf{K} & =\mathbf{U V}^{-1}=\tilde{\mathbf{V}}^{-1} \tilde{\mathbf{U}}
\end{aligned}
$$

where $\mathbf{N}, \mathbf{M}, \tilde{\mathbf{N}}, \tilde{\mathbf{M}}, \mathbf{U}, \mathbf{V}, \tilde{\mathbf{U}}$ and $\tilde{\mathbf{V}} \in \mathcal{R} \mathcal{H}_{\infty}$ must satisfy the double Bezout equation Tay et al. [1997]

$$
\left[\begin{array}{ll}
\mathbf{I} & \mathbf{0} \\
\mathbf{0} & \mathbf{I}
\end{array}\right]=\left[\begin{array}{cc}
\tilde{\mathbf{V}} & -\tilde{\mathbf{U}} \\
-\tilde{\mathbf{N}} & \tilde{\mathbf{M}}
\end{array}\right]\left[\begin{array}{ll}
\mathbf{M} & \mathbf{U} \\
\mathbf{N} & \mathbf{V}
\end{array}\right]=\left[\begin{array}{ll}
\mathbf{M} & \mathbf{U} \\
\mathbf{N} & \mathbf{V}
\end{array}\right]\left[\begin{array}{cc}
\tilde{\mathbf{V}} & -\tilde{\mathbf{U}} \\
-\tilde{\mathbf{N}} & \tilde{\mathbf{M}}
\end{array}\right] \text {. }
$$

\subsection{Fault diagnosis setup}

The residual $\boldsymbol{\epsilon}$ for $\boldsymbol{\Sigma}_{\mathbf{P}}$ is given by

$$
\boldsymbol{\epsilon}=\tilde{\mathbf{M}} \boldsymbol{y}-\tilde{\mathbf{N}} \boldsymbol{u}
$$

which is the same same residual generator used with passive fault diagnosis Frank and Ding [1994]. A more detailed discussion of the applied FD setup is given in Niemann [2006]. The fault diagnosis system is given by

$$
\boldsymbol{\Sigma}_{\mathrm{FD}}:\left\{\begin{array}{l}
e=\mathbf{P}_{\mathrm{ed}}(\boldsymbol{p}) \boldsymbol{d}+\mathbf{P}_{\mathrm{e} \eta}(\boldsymbol{p}) \boldsymbol{\eta} \\
\epsilon=\mathbf{P}_{\epsilon \mathrm{d}}(\boldsymbol{p}) d+\mathbf{P}_{\epsilon \eta}(\boldsymbol{p}) \boldsymbol{\eta}
\end{array}\right.
$$

where for the nominal system the transfer function from $\boldsymbol{\eta}$ to $\boldsymbol{\epsilon}$ is zero and a zero-mean disturbance signal $\boldsymbol{d}$ will result in a zero-mean residual signal $\boldsymbol{\epsilon}$. This can be exploited to determine whether or not the observed system is in its nominal state or if $\boldsymbol{p} \neq \mathbf{0}$.

\subsection{Evaluation of residual signals}

The injection of a known signal into the system either the auxiliary signal $\boldsymbol{\eta}$ via the control signal $\boldsymbol{u}=\tilde{\mathbf{V}}^{-1}(\tilde{\mathbf{U}} \boldsymbol{y}+\boldsymbol{\eta})$ or if the disturbance signal $\boldsymbol{d}$ is known, can be used to investigate the observer-based residual signal $\boldsymbol{\epsilon}$. If e.g. a sinusoidal signal with the frequency $\omega$ is injected into the system, two new signal can be formed from the residual signal

$$
\boldsymbol{c}=\boldsymbol{\epsilon} \cos (\omega t) \text { and } \boldsymbol{s}=\boldsymbol{\epsilon} \sin (\omega t)
$$

The signals $c$ and $s$ can then examined by various detection algorithms. A simple detection algorithm is the CUSUM test in a two-sided version

$$
\boldsymbol{z}_{k+1}=\max \left(\boldsymbol{z}_{k}+\frac{\tau_{k}}{\sigma}\left[\begin{array}{c}
\boldsymbol{c} \\
-\boldsymbol{c} \\
\boldsymbol{s} \\
-\boldsymbol{s}
\end{array}\right]-\frac{\gamma}{2}, 0\right)
$$

where $\tau_{k}$ is a time step scaling factor to be explained in the next subsection, $\sigma$ is the standard deviation of $c$ and $s$ and $\gamma$ is a tuning parameter.

\subsection{The wind turbine example}

The wind turbine is already subjected to a disturbance signal which can be used instead of an actively injected signal. As the wind turbine rotates, the blades alternate between high and low wind speeds, which are effects of the spatially distributed turbulent wind field and by the wind shear caused by the ground friction, making the wind speeds nearer ground lower than wind speeds at higher altitudes.

The rotor varies more or less with wind speed depending on the mode of operation. At partial load, below rated wind speed, the wind turbine controller attempts to maximize the power capture by keeping the ratio between wind speed and rotor speed at its optimum value. Thus the rotor speed varies and the disturbance signal injected into the system varies in frequency. The rotor azimuth angle $\phi$ can be used as input to the two residual derived signals

$$
\boldsymbol{c}=\boldsymbol{\epsilon} \cos (\phi) \text { and } \boldsymbol{s}=\boldsymbol{\epsilon} \sin (\phi)
$$

The variation of frequency can instead be considered as a constant frequency with time steps of varying length and the CUSUM detection algorithm should be corrected by a time step correction factor

$$
\tau=\Omega_{\text {nom }} / \Omega \text {. }
$$

\section{RESULTS}

Simulations have been performed in the multi-body aeroservo-elastic software HAWC2 Larsen and Hansen [2007] developed by Ris $\varnothing$ DTU. The presented simulation is performed with a mean wind speed of $8 \mathrm{~m} / \mathrm{s}$ and the wind turbine operate thus only in partial load conditions. A power law wind shear with coefficient of 0.14 and a Mann (see Mann [1998]) turbulence with turbulence intensity of 0.14 as well as a potential flow tower shadow model are used in the simulation. The wind turbine used in the simulation is the $5 \mathrm{MW}$ reference wind turbine defined in Jonkman et al. [2009].

Two simulations are presented: A nominal simulation and faulty simulation. In the faulty simulation, blade 1 have been mounted with an 1 degree offset. This fault can also be seen as an example of changed aerodynamic properties of blade 1 caused by e.g. surface pollution, icing, aerodynamic profile production tolerances etc.

The extended Kalman filter presented in Section 4 is used as the residual generator and several options for selecting the residual to investigate exists. The output error or innovation $\boldsymbol{v}_{k}=\boldsymbol{y}_{k}-\hat{\boldsymbol{y}}_{\boldsymbol{k}}$ of appropriate sensors, e.g. blade root moment sensors. Another option, and the one presented in this section, is the estimated state noise $\boldsymbol{w}_{k}=\mathbf{L}_{k} \boldsymbol{v}_{k}$. In particular the wind speed estimate is investigated.

Fig. 1 depicts the wind speed estimates in the nominal and faulty case compared to point wind speeds measured at 70 percent of the blade radii, which is typically considered to representative for the wind speeds felt by the entire 
blade. It can be seen that the wind speed estimates follow the trends of the real wind speed, but have a small offset due to the fact, that control design model used by the extended Kalman filter does not accurately describe the wind turbine model implemented in $\mathrm{HAWC} 2$, a significant difference is blade flexibility not included in the control design model.

In Fig. 2, the residuals of wind speed estimates in fixed frame coordinates multiplied with $\cos \phi$ and $\sin \phi$, respectively, can be seen. It can be seen that in the first coordinate $V_{0}$ (Fig. 2(a)) it is hard to distinguish the nominal case from the faulty case. The fact that the rotor speed frequency is not a significant component in the signal, suggests that the perturbation caused by the fault is more of an additive than multiplicative nature. In the two other signals (Fig. 2(b) and 2(c)) the nominal and faulty case are easier to distinguish and those two signals should be used for fault detection.

Fig. 3 shows the results of a CUSUM test applied to the signals seen in Fig. 2 and shows that the two signals seen in Fig 3(b) and 3(c) are clearly good candidates for fault detection signals.

At last, it should be pointed out that other types of rotor faults could be considered. It is possible to detect all different rotor faults mentioned in Section 1.

\section{CONCLUSION}

The presented method, based on an observer-based residual generator, is able to detect asymmetries in a wind turbine rotor. The multi-blade coordinate transformation has been shown to render the nominal case residuals as zero-mean signals when multiplied examined with regards to the frequency of the injected signal. Future work entails investigating other faults and possibly development of fault tolerant controllers.

\section{REFERENCES}

G. Bir. Multi-blade coordinate transformation and its application to wind turbine analysis. In 46th $A I A A$ aerospace sciences meeting and exhibit, Reno, NV., 2008. [CD ROM].

P. Caselitz and J. Giebhardt. Rotor condition monitoring for improved operational safety of offshore wind energy converters. Journal of Solor Energy Engineering, 127 (2):253-261, 2005. ISSN 15288986.

R. P. Coleman and A. M. Feingold. Theory of self-excited mechanical oscillations of helicopter rotors with hinged blades. Technical Report 1351, National Advisory Committee for Aeronautics (NACA), 1958.

P. M. Frank. Analytical and qualitative model-based fault diagnosis-a survey and some new results. European Journal of Control, 2(1):6-28, 1996. ISSN 14355671.

P. M. Frank and X. Ding. Frequency domain approach to optimally robust residual generation and evaluation for model-based fault diagnosis. Automatica, 30(5):789 804, 1994. ISSN 00051098.

Z. Hameed, Y.S. Hong, Y.M. Cho, S.H. Ahn, and C.K. Song. Condition monitoring and fault detection of wind turbines and related algorithms: A review. Renewable \& sustainable energy reviews, 13(1):1-39, 2009. ISSN 13640321.
M. H. Hansen. Improved modal dynamics of wind turbines to avoid stall-induced vibrations. Wind Energy, 6(2): 179-195, 2003. ISSN 10991824.

L. C. Henriksen and N. K. Poulsen. An online relinearization scheme suited for model predictive or linear quadratic control. IMM-Technical Report 201013, Dept. of Informatics and Mathematical Modelling, Technical University of Denmark, 2010.

L. C. Henriksen, H. H. Niemann, and N. K. Poulsen. Detecting asymmetries in the rotor of a wind turbine using the multi-blade coordinate transformation. In Diagnostics of Processes and Systems, Zamosc, Poland, 2011a.

L. C. Henriksen, N. K. Poulsen, and H. H. Niemann. Constraint handling within a multi-blade coordinate framework of a wind turbine. In 50th IEEE Conference on Decision and Control and European Control Conference, Orlando, Florida, 2011b. IEEE. To be presented.

J. Jonkman, S. Butterfield, W. Musial, and G. Scott. Definition of a 5-MW reference wind turbine for offshore system development. Technical Report NREL/TP-50038060, National Renewable Energy Laboratory, 1617 Cole Boulevard, Golden, Colorado 80401-3393, February 2009.

T. J. Larsen and A. M. Hansen. How 2 HAWC2, the user's manual. Technical Report Ris $\varnothing-\mathrm{R}-1597$ (ver. 3-1)(EN), Ris $\varnothing$ National Laboratory, 2007.

J. Mann. Wind field simulation. Probabilistic Engineering Mechanics, 13(4):269-282, 1998. ISSN 02668920.

H. H. Niemann. A setup for active fault diagnosis. IEEE Transactions On Automatic Control, 51(9):1572-1578, 2006. ISSN 15582523.

H. H. Niemann and N. K. Poulsen. Information based fault diagnosis. In Proceedings of the 17th World Congress, pages 8890-8895, Seoul, South Korea, 2008. International Federation of Automatic Control (IFAC).

P. F. Odgaard and J. Stoustrup. Unknown input observer based scheme for detecting faults in a wind turbine converter. In 7th Symposium on Fault Diagnosis, Supervision and Safety of Technical Processes, pages 161166, Barcelona, Spain, 2009. International Federation of Automatic Control (IFAC).

P. F. Odgaard and J. Stoustrup. Unknown input observer based detection of sensor faults in a wind turbine. In IEEE International Conference on Control Applications Part of 2010 IEEE Multi-Conference on Systems and Control, pages 310-315, Yokohama, Japan, 2010.

P. F. Odgaard, J. Stoustrup, R. Nielsen, and C. Damgaard. Observer based detection of sensor faults in wind turbines. In Online Proceedings of the European Wind Energy Conference, Marseille, France, 2009. European Wind Energy Association (EWEA).

N. K. Poulsen and H. H. Niemann. Active fault diagnosis - a stochastic approach. In 7th Symposium on Fault Diagnosis, Supervision and Safety of Technical Processes, pages 603-608, Barcelona, Spain, 2009a. International Federation of Automatic Control (IFAC).

N. K. Poulsen and H. H. Niemann. Active fault isolation and estimation. In Diagnosis of Processes and Systems, pages 151-158, Gdansk, Poland, 2009b.

T. T. Tay, I. M. Y. Mareels, and J. B. Moore. High Performance Control. Birkhäuser Boston, 1st edition, September 1997. 
- Real - Nominal - Fault
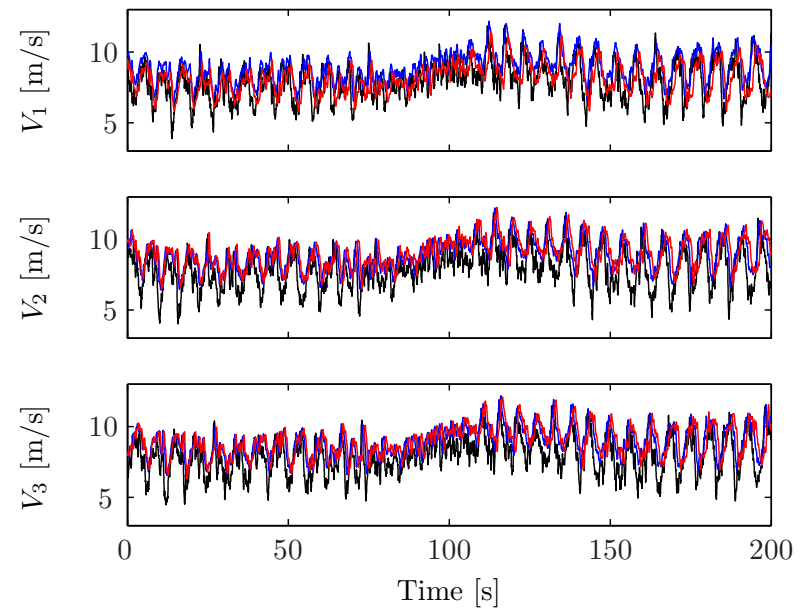

(a) Rotating frame coordinates.

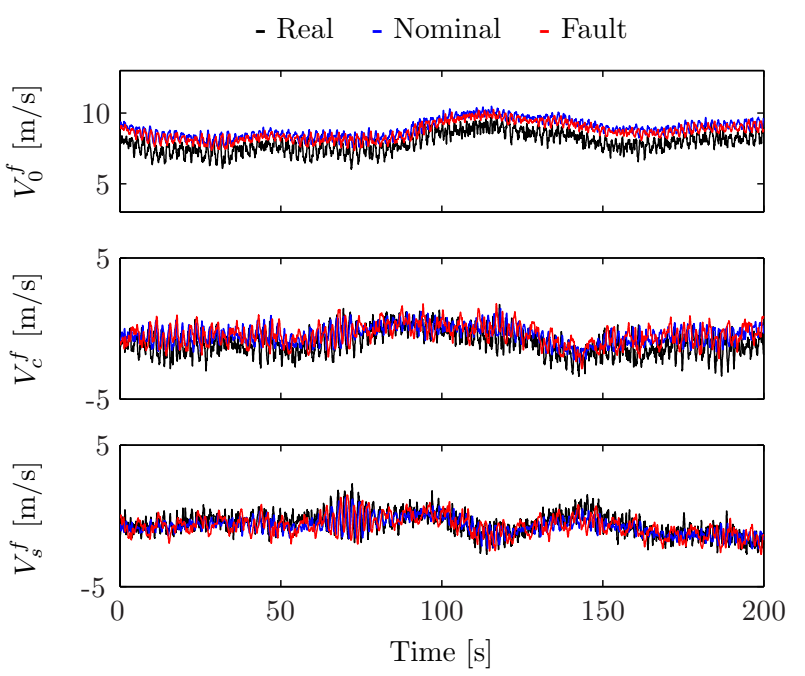

(b) Fixed frame coordinates.

Fig. 1. Wind speed estimation in the nominal and faulty case, compared to point wind speeds at 70 percent radius of blades, with the effect of tower shadow included in the signal.

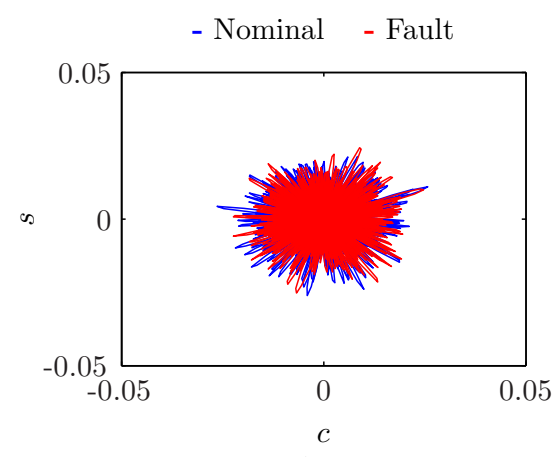

(a) $\hat{V}_{0}$

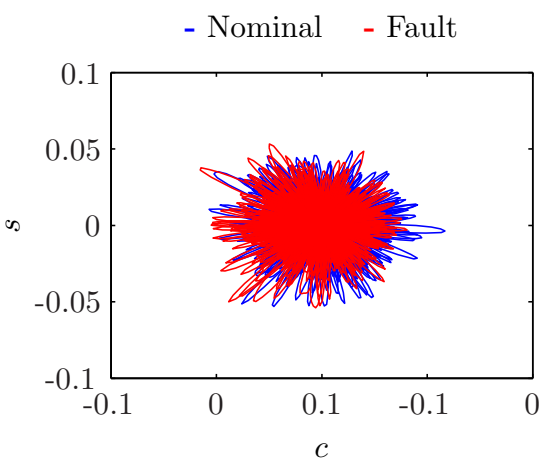

(b) $\hat{V}_{c}$

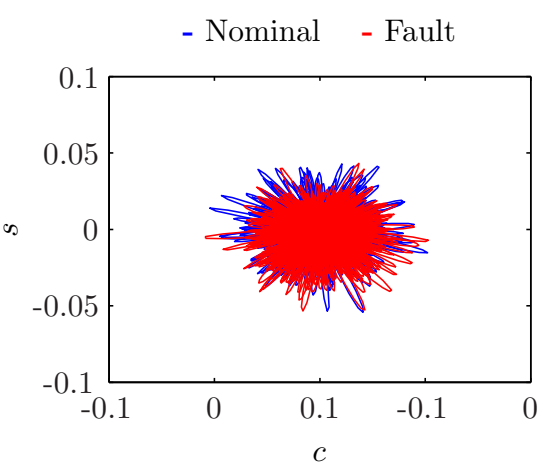

(c) $\hat{V}_{s}$

Fig. 2. Residuals of wind speed estimates in fixed frame coordinate multiplied with $\cos \phi$ and $\sin \phi$, respectively.

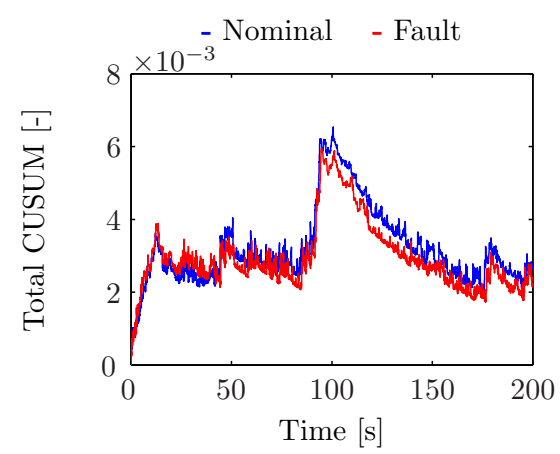

(a) $\hat{V}_{0}$

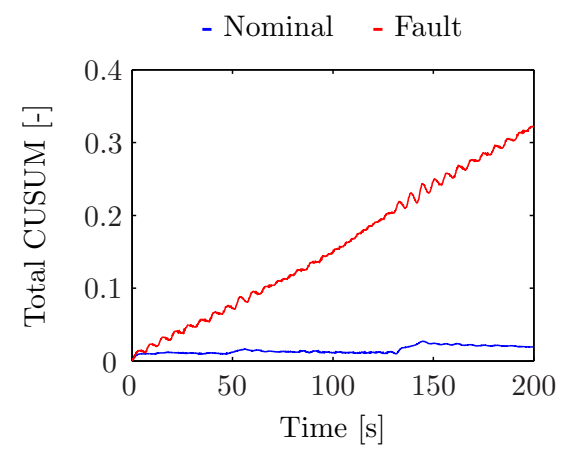

(b) $\hat{V}_{c}$

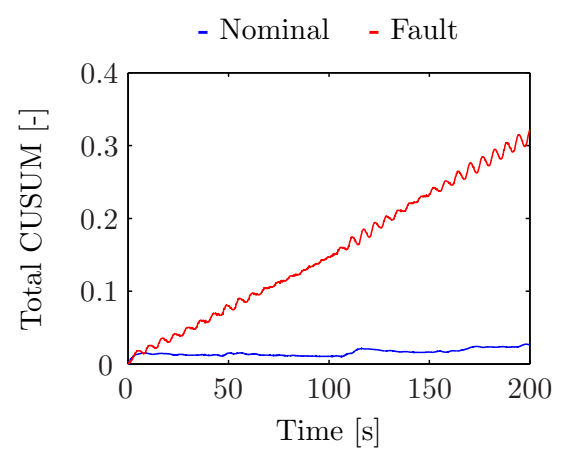

(c) $\hat{V}_{s}$

Fig. 3. Euclidean norm of CUSUM test outputs $\sqrt{\boldsymbol{z}^{T} \boldsymbol{z}}$

C.-S. Tsai, C.-T. Hsieh, and S.-J. Huang. Enhancement of damage-detection of wind turbine blades via cwt-based approaches. IEEE Transactions On Energy Conversion, 21(3):776-781, 2006. ISSN 15580059.

S. J. Watson, B. J. Xiang, W. Yang, P. J. Tavner, and C. J. Crabtree. Condition monitoring of the power output of wind turbine generators using wavelets. IEEE Transactions On Energy Conversion, 25(3):715-721, 2010. ISSN 15580059 .
$\mathrm{X}$. Wei and M. Verhaegen. Sensor and actuator fault diagnosis for wind turbine systems by using robust observer and filter. Wind Energy, 14(4):491-516, 2011.

X. Wei, M. Verhaegen, and T. van den Engelen. Sensor fault diagnosis of wind turbines for fault tolerant. In Proceedings of the 17th World Congress, pages 32223227, Seoul, South Korea, 2008. International Federation of Automatic Control (IFAC). 